\title{
Hierarchical Optimization for Neutron Scattering Problems
}

\author{
Feng Bao, Rick Archibald \\ Computer Science and Mathematics Division, Oak Ridge National Laboratory, One Bethel Valley Road, P.O. Box 2008, \\ MS-6211, Oak Ridge, TN 37831-6211 \\ Dipanshu Bansal, Olivier Delaire \\ Materials Science and Technology Division, Oak Ridge National Laboratory, One Bethel Valley Road, P.O. Box 2008, \\ MS-6118, Oak Ridge, TN 37831-6118
}

\begin{abstract}
We present a scalable optimization method for neutron scattering problems that determines confidence regions of simulation parameters in lattice dynamics models used to fit neutron scattering data for crystalline solids. The method uses physics-based hierarchical dimension reduction in both the computational simulation domain and the parameter space. We demonstrate for silicon that after a few iterations the method converges to parameters values (interatomic force-constants) computed with density functional theory simulations.
\end{abstract}

Keywords: Neutron scattering, model reduction, global optimization, stochastic, confidence distribution

\section{Introduction}

Neutron scattering is a powerful technique for the experimental measurement of the atomic structure and dynamics of condensed matter [3, 1, 2. It has been widely used in crystallography, physics, physical chemistry, biophysics, and materials research. Neutron scattering experiments probe the sample's scattering function, $S(\boldsymbol{Q}, \omega)$, also called dynamical structure factor [3, 1, 2]. In the case of nuclear scattering (neutrons can also be scattered by magnetic spins), $S(\boldsymbol{Q}, \omega)$ contains information about the spatial arrangements of atoms inside the sample, as well as the types of motions followed by the atoms [4, 5. In most cases of crystalline matter, the atoms are arranged periodically in space with a repeated unit cell, and the atoms undergo small oscillations in time around their equilibrium positions, with a motion that can be expanded in a set of harmonic oscillators (so-called phonons). The interatomic distances are of the order of Angstroems and the vibrational frequencies for the atomic oscillations are of the order of terahertz. These collective oscillations of atoms on the crystalline lattice have a scattering function, $S(\boldsymbol{Q}, \omega)$, whose support is confined to the so-called phonon dispersion surface in four dimensional $(\boldsymbol{Q}, \omega)$ space, where $\boldsymbol{Q}$ denotes the three dimensions of momentum space and $\omega$ denotes the energy transfer between neutron and sample. What can be experimentally measured in a neutron scattering experiment is the scattered intensity which is directly proportional to the scattering function, $S$, convolved with the response of the experimental apparatus (resolution function, $R$ ), and detailed in the next section. 
The scattered intensity convolution is computationally expensive in four dimensional $(\boldsymbol{Q}, \omega)$ space, and mathematical research has made some relatively recent strides in convolution integration that can be adapted by neutron scientists to build fast and accurate approximations to neutron scattering functions. These advancements include analytic evaluation of Gaussian by Gaussian convolutions [6, 7], fast algorithms for inverse transforms of sums of Gaussians [8, fast Gaussian transform [9, 10, compressed sensing of kernels [1], and limited memory block Krylov singular value decomposition (LMSVD) [12. There exists a strong need today to develop mathematical methods that can determine the optimal set of force-constants parameters $\{\Phi\}$, which best reproduces the neutron intensity measurements, and we denote $S_{\{\Phi\}}$ to be the scattering function related to the force-constants parameters $\{\Phi\}$. Such methods would have a broad impact maximizing scientific returns from neutron scattering experiments. However, constructing a computational efficient method to extract $\{\Phi\}$ from the neutron scattering intensity is difficult because the parameter space has a large dimension, $\operatorname{card}\{\Phi\} \sim 50$, and because of noise in the experimental data, as well as often incomplete information about the resolution function $R$. The focus of this research is on scalable methods for this high dimensional parameter space.

In this paper, we consider the neutron scattering problem as an inverse optimization problem and focus on inelastic neutron scattering from phonons in harmonic crystalline materials. Under the framework of this type of scattering problem, there are several difficulties that make conventional optimization methods difficult. First of all, this is a high dimensional optimization problem, thus the computational cost is very high. Also, there are multiple local optima in the problem and global optimization algorithm is needed. In addition, the scattering intensity measurement is a large data set and comparing theoretical results with the entire intensity data is expensive. To deal with these difficulties, we develop a scalable hierarchical optimization algorithm which is composed by two parts: a physics-based model reduction method that decomposes a high dimensional optimization problem into several lower dimensional suboptimization problems; a hierarchical searching algorithm which is a stochastic global optimization method using only a portion of the intensity measurement.

The rest of this paper is organized as follows. In the next section, we give a brief description to the neutron scattering optimization problem. In Section 3, we introduce our optimization algorithm to solve the neutron scattering problem. In Section 4, we show an example to indicate the effectiveness of our algorithm. Finally, Section 5 contains conclusions and directions for the future research.

\section{Neutron Scattering Optimization Problem}

In the neutron scattering optimization problem, we aim to determine a model scattering function $S$ which provides details about the physical properties of the studied material, given the experimental neutron intensity $I(\boldsymbol{Q}, \omega)$. The intensity measured $I(\boldsymbol{Q}, \omega)$ spans a four dimensional domain in $Q_{x}, Q_{y}, Q_{z}$, and $\omega$, where $\boldsymbol{Q}$ denotes the three dimensional neutron wave vector transfer, probing spatial periodicities in the sample, and $\omega$ is the energy transfer between neutron and sample. Such $4 \mathrm{D}$ datasets are efficiently 
collected with state-of-the-art experiments on single-crystalline samples with time-of-flight spectrometers [14, 15, 16], such as the ARCS, CNCS, or HYSPEC spectrometers at the Spallation Neutron Source at Oak Ridge National Laboratory [17, 18]. The experimental resolution function, $R$, which contains characteristics of the instrument and is independent of the sample material, can play a crucial role in detailed interpretation and understanding of the results, and must be convoluted with the scattering function.

The mathematical and computational challenges of neutron scattering center around the recovery of the scattering function $S$, based on an experimentally-measured intensity $I=S * R$. Since recovery of $S$ from $I$ is a non-linear problem, we use an inverse algorithm, minimizing the deviation between a model intensity $I_{\{\Phi\}}$ and the measured intensity $I$ :

$$
\min _{\sigma_{S}}\left\{\left\|I_{\{\Phi\}}-I\right\|_{L^{2}}^{2}\right\}
$$

where $\{\Phi\}$ is a set of optimizable parameters specifying the physics of the sample and $\sigma_{S}$ is the set of scattering functions related to $\{\Phi\}$.

In this research, we focus on the scientifically important problem of using neutron scattering experiments to extract information about the microscopic dynamics of atoms in materials. In this scientific case, the parameters $\{\Phi\}$ are used to describe the spring stiffnesses (force-constants) between atoms in the sample, which control the atomic vibrations (or "phonons"). However, determining these force-constants $\{\Phi\}$ from the neutron scattering intensity is a challenging problem because of the large number of unknown force-constants, and because of the four dimensional convolution of $S$ with the instrumental resolution $R$.

The model intensity $I_{\{\Phi\}}$ is obtained by building a model of the scattering function for phonons in the crystal, $S_{\{\Phi\}}$, from a set of force-constant parameters $\{\Phi\}$, and convoluting it with the instrument resolution:

$$
I_{\{\Phi\}}(\boldsymbol{Q}, \omega)=S_{\{\Phi\}}(\boldsymbol{Q}, \omega) * R(\boldsymbol{Q}, \omega) .
$$

Thus, we solve for $S_{\{\Phi\}}$ via optimization of the force-constants $\{\Phi\}$ in the lattice dynamics model, which speficies $S_{\{\Phi\}}$. Assuming a potential energy that is quadratic in the atomic positions $\boldsymbol{r}$, we construct $S$ from the set of interatomic force-constants, $\Phi_{i, j}$, between pairs of atoms $\left(\boldsymbol{r}_{i}, \boldsymbol{r}_{j}\right)$. Fourier transformation of these position-dependent force-constants, $\Phi_{i, j}\left(\boldsymbol{r}_{j}-\boldsymbol{r}_{i}\right)$, leads to the construction of a wavevector-dependent dynamical matrix, $\mathcal{D}$ (hermitian, positive definite). The eigenvalues of $\mathcal{D}$ are the squares of atomic vibration frequencies, $\omega^{2}$, corresponding to different modes of vibrations (phonons), and the corresponding eigenvectors provide the spatial pattern of atomic vibration in each mode ("polarization vectors", $\boldsymbol{\epsilon}$ ) [4].

In the present study, the model scattering function $S_{\{\Phi\}}$ is constructed as follows:

$$
S_{\{\Phi\}}(\mathbf{Q}, \omega) \propto \sum_{s, \mathbf{q}} \frac{\left\langle n_{s}+1\right\rangle}{\omega_{s}^{\{\Phi\}}}\left|\sum_{d}\left(\mathbf{Q} \cdot \epsilon_{d s}^{\{\Phi\}}\right)\right|^{2} \times \delta\left(\omega-\omega_{s}^{\{\Phi\}}(\boldsymbol{q})\right) \delta(\mathbf{Q}-\mathbf{q})
$$

where $s$ and $d$ denotes the phonon branch index and atom index in the unit cell, respectively, and $\left\langle n_{s}+1\right\rangle$ 
is the thermal occupation factor $\left(n_{s, q}\right.$ the Bose-Einstein distribution for mode $\left.(s, q)\right)$. In equation 3 , the only quantities that depend on the force-constant parameters $\{\Phi\}$ are the phonon frequencies $\omega_{s}^{\{\Phi\}}$ and eigenvectors $\epsilon_{d s}^{\{\Phi\}}$. The delta functions assure conservation of energy and momentum in the scattering process, so the intensity is localized on $3 p$ surfaces $\omega_{1 \leqslant s \leqslant 3 p}^{\{\Phi\}}(\boldsymbol{Q})$ in $(\boldsymbol{Q}, \omega)$ space ( $p$ is the number of atoms in the primitive cell), and the so-called branch index $s$ labels each of these surfaces. For a given set of parameter values $\{\Phi\}$, we build-up a force-constant tensor $\left[\Phi_{x, y, z, i, j}\right]$ and compute the dynamical matrix $\mathcal{D}=\mathcal{F}[\Phi]$ as the Fourier transform of $[\Phi]$, and then diagonalize it to obtain the phonon eigenvectors $\epsilon_{d s}$ and eigenvalues $\omega_{s}^{2}$. The computation of $S_{\{\Phi\}}$ given $\{\Phi\}$ was performed using the software SIMPHONIES 19 .

In this paper, we aim to find parameters that determine interatomic force-constants $\{\Phi\}$, denoted by a parameter vector $\boldsymbol{\xi}=\left(\xi_{1}, \xi_{2}, \cdots, \xi_{N}\right) \in \mathbb{R}^{N}, N \in \mathbb{N}$, which minimize the distance defined in (1).

\section{Optimization Algorithm}

Now we present our hierarchical optimization algorithm to find the optimal $\boldsymbol{\xi}$, an $N$-dimensional optimization procedure. However when the dimension $N$ is large, the optimization procedure becomes very difficult and expensive. To address the challenge of high dimensional optimization, in Section 3.1 we introduce a model reduction method which partitions the $N$-dimensional vector $\boldsymbol{\xi}$ into several groups. In this way, the original optimization problem is decomposed into several sub-optimization problem and we only solve one sub-optimization problem for one group of parameters at each time. Then in Section 3.2 , we focus on one sub-optimization problem with one group of parameters and develop an optimization algorithm which solves for the global optimal in a hierarchical framework.

\subsection{Model reduction}

We first introduce a model reduction method which reduces the number of parameters we need to optimize at each time. According to the physical structure of the material, we can partition all the parameters in $\boldsymbol{\xi}$ into several subvectors $\boldsymbol{\xi}_{p}$, such that $\boldsymbol{\xi}=\left(\boldsymbol{\xi}_{1}, \cdots, \boldsymbol{\xi}_{p}, \cdots, \boldsymbol{\xi}_{P}\right), P \leq N$. Each subvector $\boldsymbol{\xi}_{p}$ contains parameters which define the interaction between the pair of atoms. Interactions between pairs of atoms $(i, j)$ tend to decrease as a function of the physical distance between those atoms in the crystal, $\left|\boldsymbol{r}_{i}-\boldsymbol{r}_{j}\right|$. Hence, we group our parameters such that one individual group $\boldsymbol{\xi}_{p}$ represent the interaction between the pair of atoms for a fixed separation. Subscript $p$ in $\boldsymbol{\xi}_{p}$ directly correlates with distance between the pair of atoms, and increases with increase in interatomic separation between the pair of atoms. For example, $\boldsymbol{\xi}_{1}$ corresponds to first nearest neighbor interatomic force parameters, $\boldsymbol{\xi}_{2}$ corresponds to second nearest neighbor interatomic force parameters, and so on. Apparently, the pairs of atoms with closer distances have stronger interactions and therefore the corresponding interatomic force parameters are more important. To better illustrate our model reduction method, we use figure 1 to show the relation between interatomic interactions and atoms. With this definition, when index $p$ becomes larger and larger, we are 
including interaction between pair of atoms that are far apart, and by inclusion of additional interatomic interactions, our approximation of $S_{M}$ will approach towards experimental observations. In this connection,
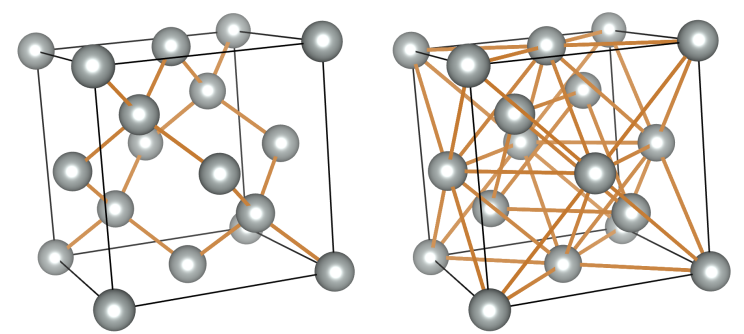
First and second nearest neighbor interactions

$\xi_{1}=\left(\xi_{1}, \xi_{2}, \xi_{3}, \xi_{4}, \xi_{5}\right)$

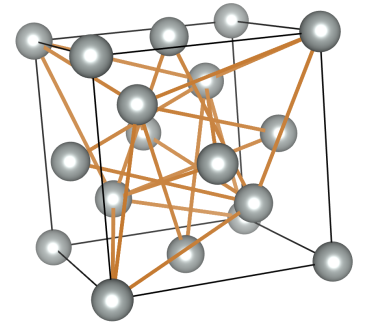

Third nearest

neighbor

interactions

$\xi_{2}=\left(\xi_{6}, \xi_{7}\right)$

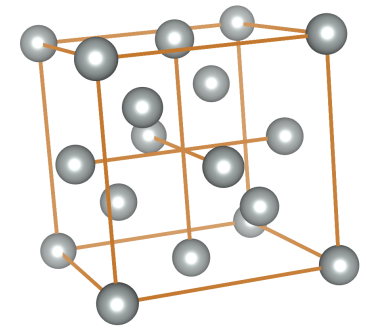

Fourth nearest

neighbor

interactions

$\xi_{3}=\left(\xi_{8}, \xi_{9}\right)$

Figure 1: Illustration of Silicon crystal structure with first to fourth nearest neighbor interaction. Here $\xi_{i}$ represent the force constant $\{\Phi\}$ parameter between nearest neighbor atoms, which are grouped into $\boldsymbol{\xi}_{p}$. We should note here that in this illustration we have combined first and second nearest neighbor interactions into one parameter $\boldsymbol{\xi}_{1}$, while third and fourth nearest neighbor interactions have been represented with $\boldsymbol{\xi}_{2}$, and $\boldsymbol{\xi}_{3}$, respectively. Based on symmetry operations for different nearest neighbor pairs, each group $\boldsymbol{\xi}_{p}$ contains varying number of independent force constant parameters. Each $\xi_{i}$ corresponds to independent force constant $\{\Phi\}$ parameter required to describe given nearest neighbor interatomic interactions.

instead of solving for the $N$-dimensional vector $\boldsymbol{\xi}$ directly, we solve for $\boldsymbol{\xi}_{p}, p=1,2, \cdots, P$ separately. Each $\boldsymbol{\xi}_{p}$ is a lower dimensional parameter vector and we find the optimal $\boldsymbol{\xi}_{p}$ following the increasing order of $p$ i.e. $p=1,2, \cdots, P$. When we seek the optimal $\boldsymbol{\xi}_{1}$, we fix $\boldsymbol{\xi}_{2}, \cdots, \boldsymbol{\xi}_{P}$ as their initial values and find the estimate $\tilde{\boldsymbol{\xi}}_{1}$ for the optimal $\boldsymbol{\xi}_{1}$. When we have estimates $\tilde{\boldsymbol{\xi}}_{1}, \cdots \tilde{\boldsymbol{\xi}}_{p}$, we fix $\boldsymbol{\xi}_{1}=\tilde{\boldsymbol{\xi}}_{1}, \cdots, \boldsymbol{\xi}_{p}=\tilde{\boldsymbol{\xi}}_{p}$, choose $\boldsymbol{\xi}_{p+2}, \cdots, \boldsymbol{\xi}_{P}$ as the initial values and find the estimate $\tilde{\boldsymbol{\xi}}_{p+1}$ for the optimal $\boldsymbol{\xi}_{p+1}$. In this way, we get the estimate $\tilde{\boldsymbol{\xi}}:=\left(\tilde{\boldsymbol{\xi}}_{1}, \cdots, \tilde{\boldsymbol{\xi}}_{P}\right)$ for the optimal parameter vector $\boldsymbol{\xi}$ in a recursive way and each optimization procedure is for only one segment of the vector $\boldsymbol{\xi}$ which requires fewer computational effort.

Notice that since each estimate $\tilde{\boldsymbol{\xi}}_{p}$ is based on the initial values of $\boldsymbol{\xi}_{p+1}, \cdots, \boldsymbol{\xi}_{P}$, the estimate optimal $\tilde{\xi}$ we find in this way may be inaccurate. To adjust the estimate parameter and search for a more accurate estimate for optimal $\boldsymbol{\xi}$, we introduce a looped optimization procedure. We consider the optimization process 
of getting estimate $\tilde{\boldsymbol{\xi}}$ as optimization loop $l=1$ and define $\overline{\boldsymbol{\xi}}^{1}$ by

$$
\overline{\boldsymbol{\xi}}^{1}=\left(\overline{\boldsymbol{\xi}}_{1}^{1}, \cdots \overline{\boldsymbol{\xi}}_{P}^{1}\right):=\left(\tilde{\boldsymbol{\xi}}_{1}, \cdots, \tilde{\boldsymbol{\xi}}_{P}\right), \quad \text { where } \overline{\boldsymbol{\xi}}_{p}^{1}=\tilde{\boldsymbol{\xi}}_{p}
$$

as the optimization result of the first loop. Then, in optimization loop $l, l \in \mathbb{N}$ and $l \geq 2$, we use $\overline{\boldsymbol{\xi}}^{l-1}$ to be the initial value for the parameter vector $\boldsymbol{\xi}$ and repeat the above optimization procedure to get estimate $\overline{\boldsymbol{\xi}}^{l}$ for optimal $\boldsymbol{\xi}$. When the difference between loop $l-1$ and loop $l$ is small, i.e. $\left|\overline{\boldsymbol{\xi}}^{l}-\overline{\boldsymbol{\xi}}^{l-1}\right|<\epsilon$, we consider the optimization for $\boldsymbol{\xi}$ is convergent.

\subsection{Hierarchical optimization}

In this subsection, we consider one group of parameters $\boldsymbol{\xi}_{p}, p=1,2, \cdots, P$, in a given optimization loop $l$ and develop a hierarchical optimization method focused on one sub-optimization problem to find the estimate $\tilde{\boldsymbol{\xi}}_{p}$ for optimal parameter vector $\boldsymbol{\xi}_{p}$.

\section{Stochastic hierarchical searching algorithm}

In this lattice dynamics optimization problem, there are several local optima and a global optimum for the force-constants parameter vector $\boldsymbol{\xi}_{p}$. Local optimization methods such like gradient methods and pattern search method could only find a local optimum. In this paper, we assume that the global optimal $\boldsymbol{\xi}_{p}$ lies in a large parameter space $\Omega^{p}$ and propose a global hierarchical optimization algorithm to find the optimal force-constants parameters. The central idea of this algorithm is to probe the parameter space $\Omega^{p}$ by computing the model neutron-scattering function $S_{M,\{\Phi\}}(\boldsymbol{Q}, \omega)$ for different parameters and compare with the experimental data (after convolution with $R_{M}$ ) to find the best match. If we select all the parameters in $\Omega^{p}$ and compare their simulation results with the experimental data, we can guarantee that we would find the global optimal $\boldsymbol{\xi}_{p}$. However, it is not feasible to test every parameter in the parameter space and a selection of test parameters over $\Omega^{p}$ is necessary. Choosing test parameters on the full-tensor product mesh in the parameter space is the most straightforward option. However in many situations, the dimension $d^{p}$ of the vector $\boldsymbol{\xi}_{p}$ is also large. In this case, the searching procedure is operated over a $d^{p}$-dimensional space and the number of test parameters increases exponentially which drastically increases the computational cost. To alleviate this problem, in our approach we select parameters generated by Latin hypercube sampling method (introduced in [13]) on hierarchically defined adaptive parameter spaces.

To be specific, we let the initial hierarchical parameter space be $\Omega^{p}$, i.e. $\Omega_{1}^{p}:=\Omega^{p}$. Suppose the $i$ th hierarchical parameter space $\Omega_{i}^{p}$ is given, we choose a set of test parameters by using Latin hypercube sampling method and denote them by $\left\{\boldsymbol{\xi}_{p}^{i}(j)\right\}_{j=1, \cdots, N_{p}}$, where $N_{p}$ is a given positive integer. For each test parameter $\boldsymbol{\xi}_{p}^{i}(j)$, we denote $\hat{I}\left(\boldsymbol{\xi}_{p}^{i}(j)\right)$ to be the simulation of the theoretical model given in (2) computed by using $\boldsymbol{\xi}_{p}^{i}(j)$ and $I$ is the experimental data we compare against. Then we define a weight for each test parameter by

$$
w_{i}^{p}(j):=C_{i}^{p} \exp \left(-\left\|\hat{I}\left(\boldsymbol{\xi}_{p}^{i}(j)\right)-I\right\|_{\text {error }}^{2}\right)
$$


Here, $C_{i}^{p}$ is the normalization factor such that $\sum_{j=1}^{N_{p}} w_{i}^{p}(j) / C_{i}^{p}=1$ and $\|\cdot\|_{\text {error }}$ is an error norm which measures the difference between simulation results and experimental data. In this particular optimization problem, we want to minimize the error between theoretical simulations and experimental data which are given by four dimensional matrices. However, the intensity varies in a wide range and the errors brought by high value elements in the matrix would dominate the optimization procedure. To reduce the influence of the high value elements, we define the error norm $\|\cdot\|_{\text {error }}$ as

$$
\left\|\hat{I}\left(\boldsymbol{\xi}_{p}^{i}(j)\right)-I\right\|_{\text {error }}:=\left\|\log \left(\hat{I}\left(\boldsymbol{\xi}_{p}^{i}(j)\right)+\mathbf{1}\right)-\log (I+\mathbf{1})\right\|_{L^{2}}
$$

where $\mathbf{1}$ is a four dimensional matrix of ones. In inelastic neutron scattering, there are Bragg Peaks in the intensity matrix on which we observe extremely large intensity values. To prevent extremely large values at Bragg Peaks dominating the entire error, when an element in $\hat{I}$ or $I$ is larger than a given constant $K$, we just let the value be equal to $K$.

In this way, we obtain a weight $w_{i}^{p}(j)$ for each test parameter $\boldsymbol{\xi}_{p}^{i}(j)$ in parameter space $\Omega_{i}^{p}$ and pairs $\left\{\left(\boldsymbol{\xi}_{p}^{i}(j), w_{i}^{p}(j)\right)\right\}_{j=1, \cdots, N_{p}}$ form a representation of confidence distribution of parameters in $\Omega_{i}^{p}$. This distribution includes simulation results of all the parameters $\left\{\boldsymbol{\xi}_{p}^{i}(j)\right\}_{j=1, \cdots, N_{p}}$ and the global optimal $\boldsymbol{\xi}_{p}$ lies in the high density region. We use the expectation of the confidence distribution to be our estimate optimal parameter $\tilde{\boldsymbol{\xi}}_{p}^{i}$ in $\Omega_{i}^{p}$, i.e.

$$
\tilde{\boldsymbol{\xi}}_{p}^{i}=\sum_{j=1}^{N_{p}} \boldsymbol{\xi}_{p}^{i}(j) w_{i}^{p}(j),
$$

and denote the standard deviation of the distribution by $\sigma_{i}^{p}$. Then we generate the next hierarchical parameter space $\Omega_{i+1}^{p}$ by creating a hypercube of size $\sigma_{i}^{p}$ to get a confidence region surrounding $\tilde{\boldsymbol{\xi}}_{p}^{i}$ and repeat the above procedure iteratively. When the standard deviation $\sigma_{i}^{p}$ is smaller than a threshold $\delta$, we consider the parameter space $\Omega_{i}^{p}$ is small and our estimate $\tilde{\boldsymbol{\xi}}_{p}^{i}$ is accurate enough.

Remark 1. When the hierarchical parameter space $\Omega_{i}^{p}$ is small, the influence caused by the difference in the test parameters is small. This makes the noises in the experimental data dominate the optimization procedure and weights of different parameters tend to be uniform.

\section{Data decomposition}

In our stochastic hierarchical searching algorithm for the inelastic neutron scattering optimization problem, we need to use the theoretical model to compute the numerical simulation for the scattering intensity on each test parameter and compare simulation results with the experimental data to find the confidence distribution of parameters. However, it's very expensive to compute four dimensional theoretical intensity matrices $\hat{I}$. Also, the intensity measured from a neutron scattering experiment is usually a large set of data. Thus finding the optimal parameter based on the experimental data $I$ is very difficult. To reduce the computational cost and make our algorithm more feasible for large data set $I$, we decompose the 
four dimensional data matrix into several two dimensional data matrices. We denote them by $I_{1}, I_{2}, \cdots, I_{M}$ such that $I=\cup_{m=1}^{M} I_{m}$ and refer to each two dimensional matrix as a slice of data. Given iteration index $p$ and $i$, instead of computing $\hat{I}\left(\boldsymbol{\xi}_{\boldsymbol{i}}^{p}(j)\right)$ for each test parameter $\boldsymbol{\xi}_{i}^{p}(j)$, we compute the simulation of the theoretical model on each slice, denoted by $\hat{I}_{m}\left(\boldsymbol{\xi}_{i}^{p}(j)\right)$, and compare $\hat{I}_{m}\left(\boldsymbol{\xi}_{i}^{p}(j)\right)$ with $I_{m}$. Obviously, it's much easier to find the optimal parameter based on each slice of data. The problem is how to combine optimization results on different slices together to get a global estimate. An advantage of our stochastic hierarchical optimization algorithm introduced above is we find not only the estimate for the global optimal parameter but also the confidence distribution of parameters. So at each iteration step, we would find a representation of confidence distribution over test parameters on each slice. Thus we can simply multiply all the slice-wise distributions together to get a global confidence distribution.

To be specific, for the $m$-th slice we compute the weight on parameter $\boldsymbol{\xi}_{p}^{i}(j)$ by

$$
w_{i}^{p, m}(j):=C_{i}^{p, m} \exp \left(-\left\|\hat{I}_{m}\left(\boldsymbol{\xi}_{p}^{i}(j)\right)-I_{m}\right\|_{\text {error }}^{2}\right)
$$

Here,

$$
\left\|\hat{I}_{m}\left(\boldsymbol{\xi}_{p}^{i}(j)\right)-I_{m}\right\|_{\text {error }}=\left\|\log \left(\hat{I}_{m}\left(\boldsymbol{\xi}_{p}^{i}(j)\right)+\mathbf{1}\right)-\log \left(I_{m}+\mathbf{1}\right)\right\|_{L^{2}}
$$

is the error norm, $\mathbf{1}$ is a two dimensional matrix of ones and $C_{i}^{p, m}$ is the normalization factor. The pairs $\left\{\left(\boldsymbol{\xi}_{p}^{i}(j), w_{i}^{p, m}(j)\right)\right\}_{j=1, \cdots, N_{p}}$ obtained in this way give slice-wise distributions of parameters and we consider these distributions to be independent. To combine these confidence distributions on different slices, we take the product of weights from different slices for each parameter to get a overall weight

$$
w_{i}^{p}(j):=C_{i}^{p} \prod_{m=1, \cdots M} w_{i}^{p, m}(j)
$$

where $C_{i}^{p}$ is a normalization factor. Therefore, the pairs $\left\{\left(\boldsymbol{\xi}_{p}^{i}(j), w_{i}^{p}(j)\right)\right\}_{j=1, \cdots, N_{p}}$ form a representation of confidence distribution of parameters by using the entire data set $I$ and we can estimate $\tilde{\boldsymbol{\xi}}_{p}^{i}$ and standard deviation $\sigma_{i}^{p}$.

In this data decomposition algorithm, since we estimate the confidence distribution of parameters based on each slice of data, it allows us to study more detailed properties of the material. Depending on the structure of the material measured in the experiment, different force-constants $\{\Phi\}$ (corresponding to some components of the parameter vector $\boldsymbol{\xi}$ ) are more sensitive to different slices of neutron scattering intensity data. Constructing confidence distribution of parameters based on each slice of intensity would give us more information about the relation between the parameters and a portion of data. From a certain slice of data we might get larger standard deviation in the parameter confidence distribution while another slice of data might give us smaller standard deviation. It's obvious that the larger standard deviation means the parameters are less sensitive to the data and smaller standard deviation means the parameters are more sensitive to the data. Therefore, it could be considered as a kind of sensitivity test. In Figure 2 we 
show an example of slice-wise distributions and the global distribution which illustrates what we observe in the numerical experiments that we are going to present in the next section. The blue, red and green dashed curves describe the confidence distributions of parameters based on slice 1 , slice 2 and slice 3 , respectively, and the black solid curve shows the combined overall distribution. From the figure we can see that the distribution from slice 2 has very large standard deviation, the distribution from slice 1 has smaller standard deviation and the standard deviation of the distribution from slice 3 is even smaller then slice 1. The global distribution has the smallest standard deviation among all the distributions since it's the product of all the distributions. The standard deviations of distributions from slice 1, 2 and 3 suggest the sensitivity of the parameters to different portion of data. We can see that the distribution we get from slice 2 does not give much useful information about the parameters since it's closer to a uniform distribution and errors between simulations and data are similar among parameters. However the distribution from slice 3 contributes the most to the overall distribution and parameters are more sensitive to the data in slice 3 . In this way, the distributions from different slices provide guidance in designing future neutron-scattering measurements, by informing the experimenter about which domain in the four dimensional $(\boldsymbol{Q}, \omega)$-space to measure most accurately, in order to precisely determine certain force-constants $\Phi$. Furthermore, if only a selection of slices of data ( based on the complexity of the material ) would give us an accurate confidence region to locate the optimal parameter, it's not necessary to use all the neutron scattering intensity data.

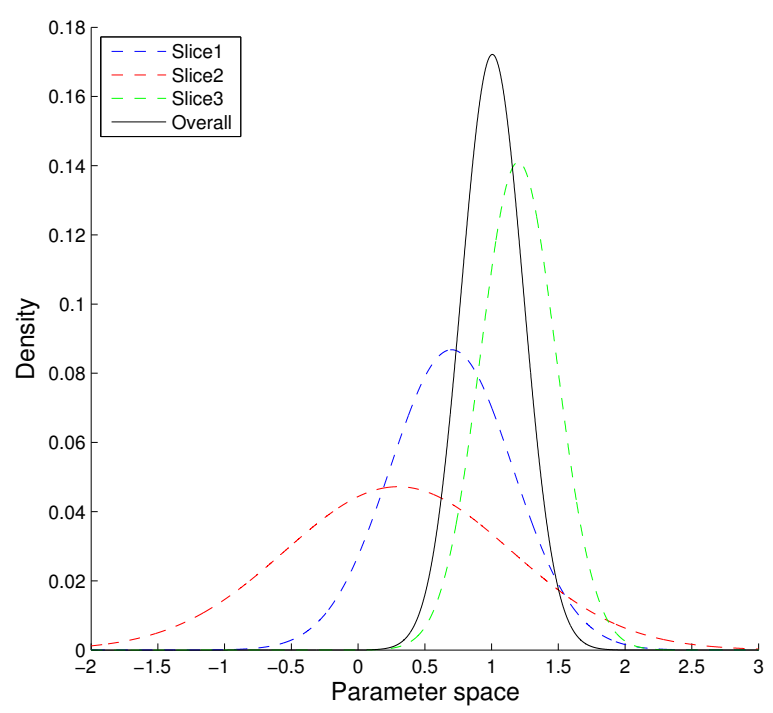

Figure 2: Illustration of distributions of slices and the overall distribution.

Remark 2. It is worth to point out that for each test parameter, the simulation of the theoretical model is computational expensive. The structure of our algorithm is scalable in computing $\hat{I}$ from different $\boldsymbol{\xi}_{p}^{i}(j)$ and this scalability makes the algorithm much more efficient to run in parallel. 


\subsection{Algorithm}

We summarize our entire algorithm in Algorithm 1

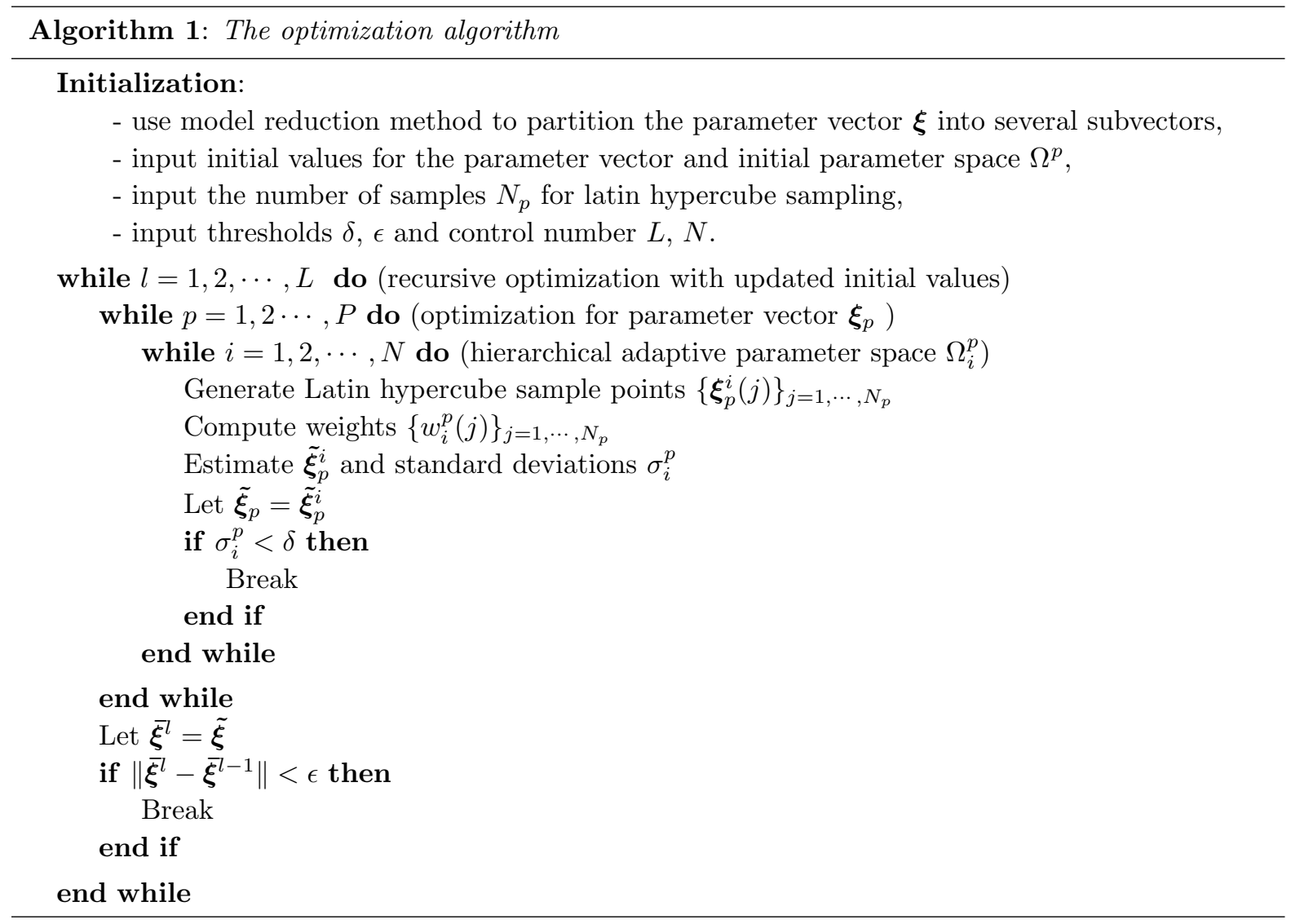

\section{Numerical experiments}

In this section, we study the material structure of silicon and present the performance of our hierarchical optimization algorithm discussed in Section 3 .

In this example, we optimize 9 parameters for silicon, i.e. $\boldsymbol{\xi}=\left(\xi_{1}, \xi_{2}, \cdots, \xi_{9}\right)$. Based on the physical structure of silicon, we let $\boldsymbol{\xi}=\left(\boldsymbol{\xi}_{1}, \boldsymbol{\xi}_{2}, \boldsymbol{\xi}_{3}\right)$, where $\boldsymbol{\xi}_{1}=\left(\xi_{1}, \xi_{2}, \xi_{3}, \xi_{4}, \xi_{5}\right), \boldsymbol{\xi}_{2}=\left(\xi_{6}, \xi_{7}\right)$ and $\boldsymbol{\xi}_{3}=\left(\xi_{8}, \xi_{9}\right)$, and consider first three nearest neighbor interatomic interactions (as shown Figure 11). For our numerical experiment, values of $\boldsymbol{\xi}$ obtained from DFT calculations can be considered as "real" parameter values. Therefore, the experimental data we use in this example are generated by simulation through the theoretical model computed using the software SIMPHONIES with the "real" parameters which are perturbed by some noises. Specifically, suppose $\bar{I}$ is the scattered intensity generated by using the DFT parameter values, the experimental data $I$ we use in this example is defined by

$$
I=\bar{I}+0.1 \gamma \cdot \bar{I}+c \beta,
$$


where $\gamma$ is a standard Gaussian noise, i.e. $\gamma \sim N(0,1)$, and the term $0.1 \gamma \cdot \bar{I}$ brings $10 \%$ of proportional noise to the intensity, $c$ is a given constant and $\beta$ is another standard Gaussian noise independent from $\gamma$ which gives the background noise. Based on the complexity of the material, we select 5 slices of two dimensional data from the four dimensional experimental intensity data matrix $I$, denoted by $I_{1}, I_{2}, \cdots, I_{5}$, and optimize over these 5 slices of data. The 5 slices are carefully chosen along high symmetry directions of reciprocal lattice structure spanning several Brillouin zones from four dimensional dataset to adequately represent the system dynamics.

We first present the behavior of hierarchical parameter spaces $\Omega_{i}^{p}$ in our optimization algorithm. To illustrate the dynamics of these spaces, we show $\Omega_{i}^{1}$ along $\xi_{1}$ direction and $\xi_{2}$ direction for 9 iteration steps in figure 3. The blue boxes are parameter spaces in the first optimization loop, i.e. $l=1$, which is the first

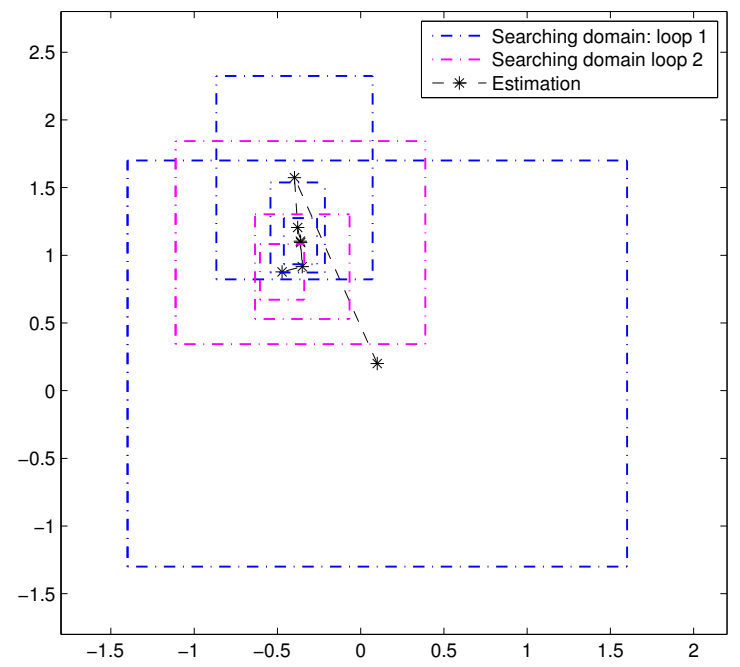

Figure 3: Hierarchical searching domains of parameter 1 and parameter 2. Blue boxes are hierarchical parameter spaces in loop 1 and magenta boxes are hierarchical parameter spaces in loop 2.

time we seek the optimal $\boldsymbol{\xi}_{1}$ before we optimize $\boldsymbol{\xi}_{2}$ and $\boldsymbol{\xi}_{3}$. The magenta boxes are parameter spaces in the second optimization loop, i.e. $l=2$, which is the optimization procedure to seek the optimal $\boldsymbol{\xi}_{1}$ after we optimize $\boldsymbol{\xi}_{2}$ and $\boldsymbol{\xi}_{3}$. The astro in the center of each box is our estimate which is the mean value of the confidence distribution of parameters in the previous searching parameter space. The biggest blue box in the figure is our initial selection of the parameter space, i.e. $\Omega_{1}^{1}$, and the astro in the center of that box is our initial guess of parameters $\xi_{1}$ and $\xi_{2}$. From the figure, we can see the convergence trend of both the estimate parameters and the searching parameter spaces.

In Figure 4 , we plot the $L^{2}$ error between the "real" parameter and the estimate parameter at each iteration step ( for different iteration indices $l, p, i$ ). The $X$-axis shows the hierarchical optimization iteration steps and the $Y$-axis shows the $L^{2}$ errors. From this figure, we can see the convergence trend of the parameters. Notice that the $L^{2}$ error does not decrease to zero since the noise in the experimental data 


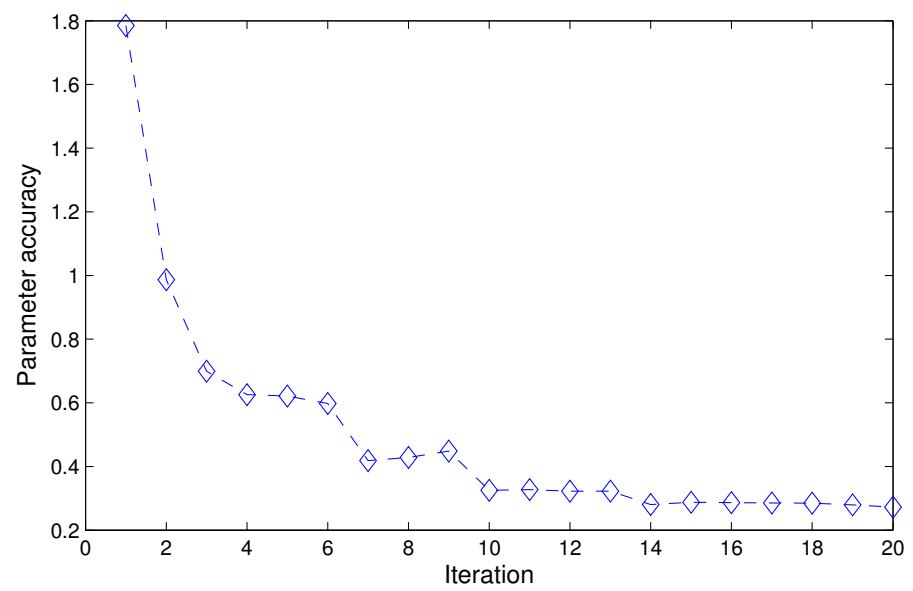

Figure 4: Parameter accuracy

brings a "barrier" between our estimate optimal parameter and the "real" parameter.

Figure 5 to Figure 10 show comparisons of experimental data and the simulation of the theoretical model intensity for slice 1, slice 2 and slice 3. In Figures 5, 6, 7 we show comparisons of two dimensional slices and the color represents the intensity. Subplot (a) in each figure is the experimental data. Subplot (b) is the simulation of the theoretical model given the initial guess of the parameters, you can see this result doesn't match the experimental data. Subplot (c) is the simulation of the theoretical model obtained by using the estimate optimal $\boldsymbol{\xi}_{1}$ in loop $l=1$ and initial guess of $\boldsymbol{\xi}_{2}$ and $\boldsymbol{\xi}_{3}$. As you can see, the simulation looks very close to the experimental data except some features. Subplot (d) is the simulation of the theoretical model obtained by using the final estimate for optimal $\boldsymbol{\xi}$ and the simulation matches the experimental data very well. From these figures we can see that in optimization loop $l=1$, although we only use initial guess values of parameters $\boldsymbol{\xi}_{2}$ and $\boldsymbol{\xi}_{2}$ when optimize the first group of parameters $\boldsymbol{\xi}_{1}$, the theoretical results give us pretty good simulations which could match experimental data well. This indicates that the first set of parameters $\boldsymbol{\xi}_{1}$ has the most substantial influence to the theoretical simulation which also demonstrates the effectiveness of our model reduction method.

To further distinguish the difference between subplot (c) and subplot (d) in each figure, we compare the intensity at certain energy levels (the cross-section of $Y$-axis at energy level $16 \mathrm{meV}, 12 \mathrm{meV}, 16 \mathrm{meV}$, respectivley) in Figures 8, 9 and 10. The blue curves are the experimental intensities; red curves are theoretical simulated intensities obtained by using the estimate optimal $\boldsymbol{\xi}_{1}$ in loop l $=1$ and initial guess of $\boldsymbol{\xi}_{2}$ and $\boldsymbol{\xi}_{3}$; the green curves give theoretical simulated intensities obtained by using the final estimate for optimal $\boldsymbol{\xi}$. From these figures, we can see the importance of optimizing parameters $\boldsymbol{\xi}_{2}$ and $\boldsymbol{\xi}_{3}$. Although from Figures 5, 6, 7, we might think the optimization for only $\boldsymbol{\xi}_{1}$ could give pretty good results, the comparison of intensities indicates that parameters $\boldsymbol{\xi}_{2}$ and $\boldsymbol{\xi}_{3}$ also play an important role, and study of these parameters is necessary. 
(a)

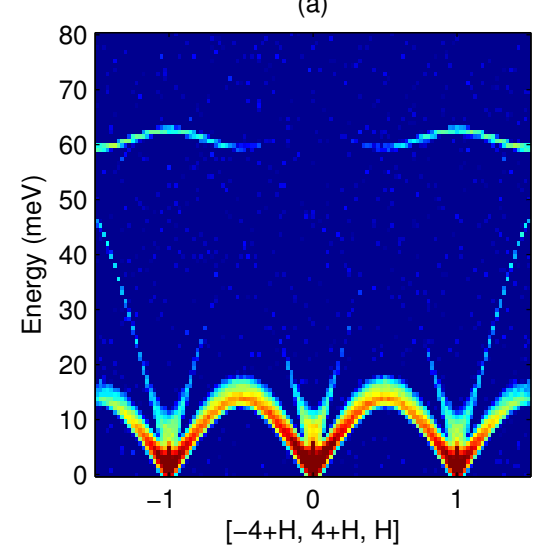

(c)

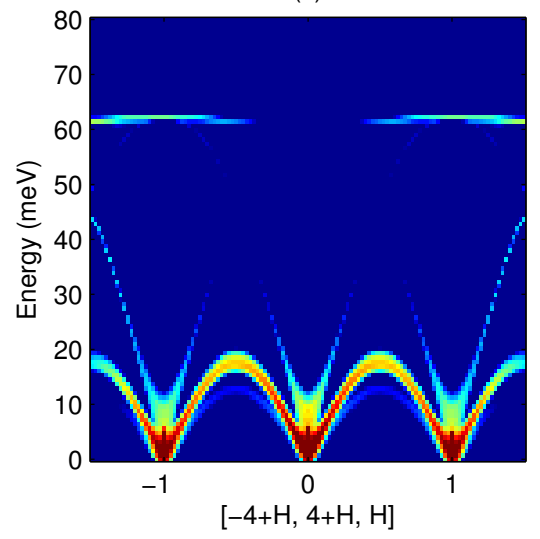

(b)

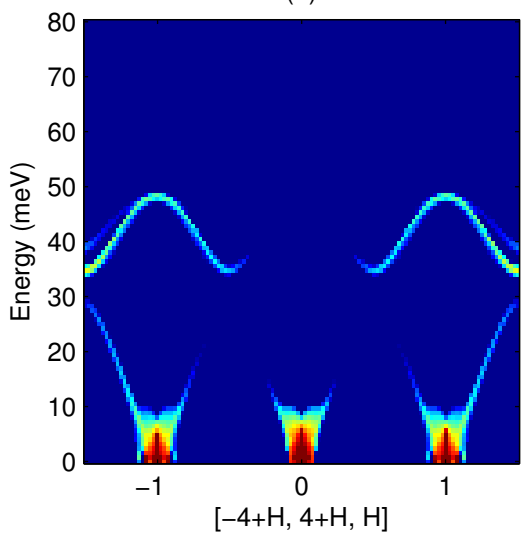

(d)

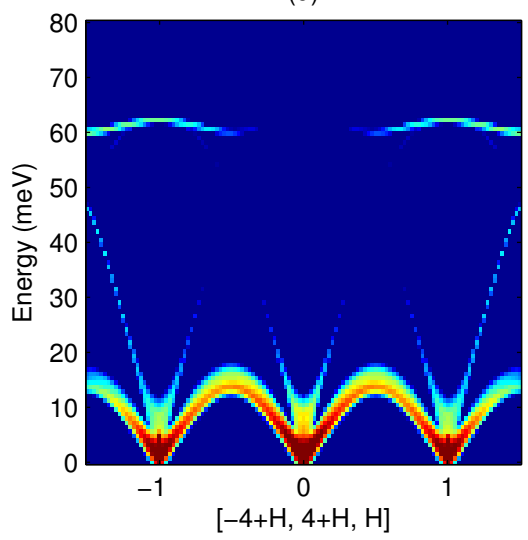

Figure 5: Images of slice 1: subplot (a) is the experimental data; subplot (b) is the simulation of the theoretical model given the initial guess of the parameters; subplot (c) is the simulation of the theoretical model after optimizing the first 5 parameters in the first optimization loop; subplot (d) is the simulation of the theoretical model obtained by using the optimal parameter. $H$ is the reciprocal lattice unit.

In Table 1 and Table 2, we present the information we learn from confidence distributions of test parameters in iteration step 8 and iteration step 16, respectively. Table 1 shows the top ranked weights of test parameters obtained from intensity data slice 1 , slice 2 and slice 3 in iteration step 8 which is an intermediate iteration step. In the same table, we also present the weights after combining all 5 slices which gives an representation of the overall confidence distribution. The "Rank" on the left column means the rank of density weights and we show the highest 15 weights we obtained from each slice and the overall weights combined all 5 slices. From this table, we can see that the decreasing rate of weights from slice 2 is very low which means the standard deviation of the distribution is large and the parameters are not sensitive to this portion of data. On the other hand, the decreasing rate of weights from slice 3 is much higher compared to slice 2 which means the standard deviation of the distribution is small and the parameters are more sensitive to this portion of data. In table 2, we present the top ranked weights of 
(a)

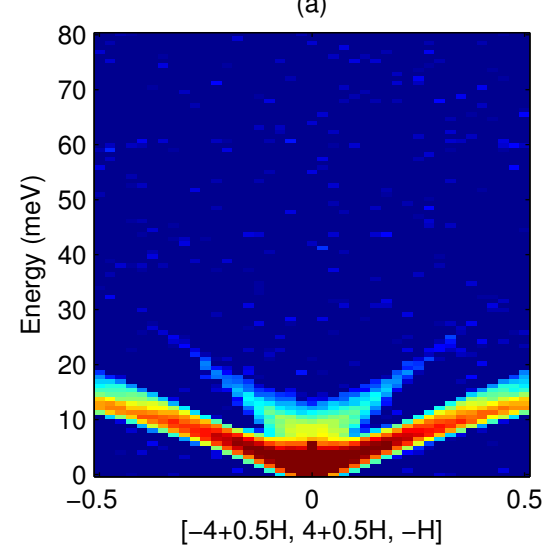

(c)

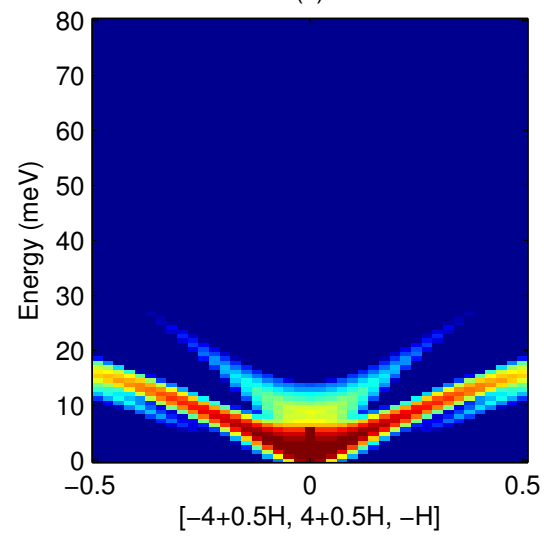

(b)

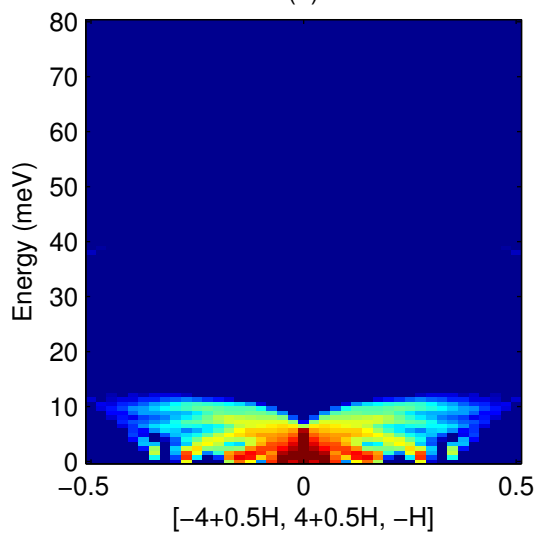

(d)

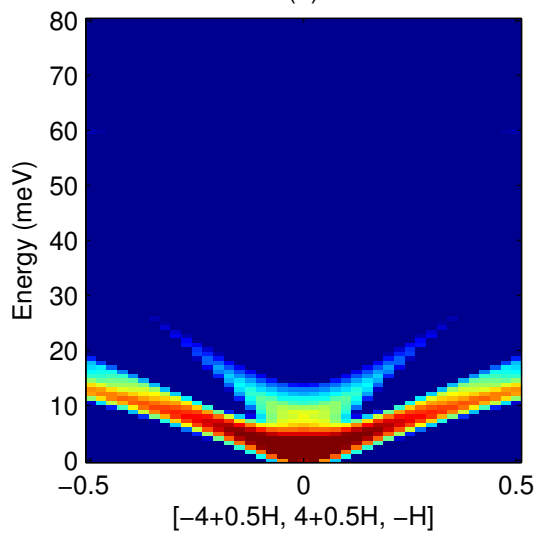

Figure 6: Images of slice 2: subplot (a) is the experimental data; subplot (b) is the simulation of the theoretical model given the initial guess of the parameters; subplot (c) is the simulation of the theoretical model after optimizing the first 5 parameters in the first optimization loop; subplot (d) is the simulation of the theoretical model obtained by using the optimal parameter. $H$ is the reciprocal lattice unit.

test parameters obtained from intensity data slice 1, slice 2 and slice 3 in the iteration step 16 and we also present the weights after combining all 5 slices. In this iteration step, the parameter space in which we construct confidence distribution is already very small. We can see that the decreasing rates of weights are low for each slice of data and distributions of parameters are similar to uniform distribution. This means many test parameters in the parameter space are equally important and the optimization is convergent.

\section{Conclusion}

In this work, we developed a scalable hierarchical global optimization algorithm to solve for the scattering function from neutron intensity measurements. Specifically, we introduced a physical structure based model reduction to decompose the original high dimensional optimization problem into several lower dimensional sub-optimization problems and solve one sub-optimization problem at each time. For each sub- 
(a)

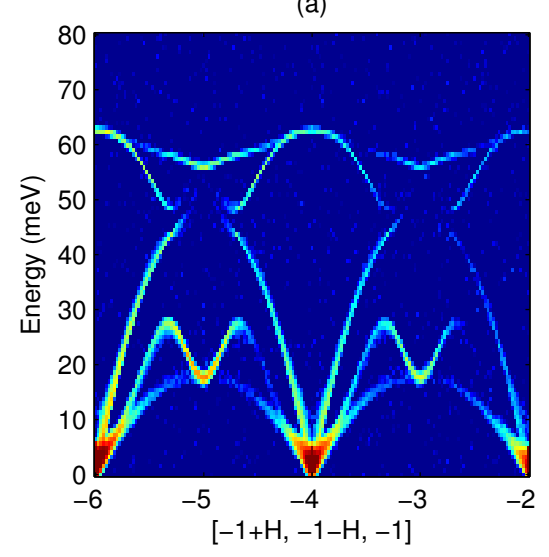

(c)

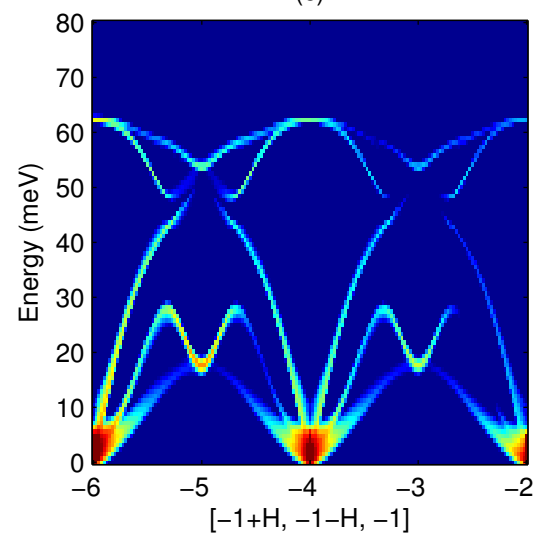

(b)

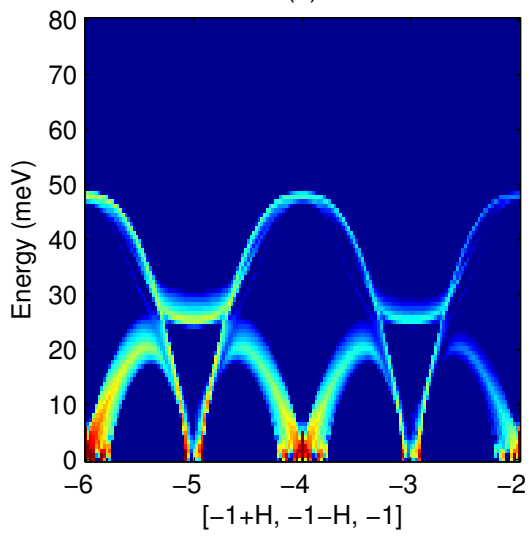

(d)

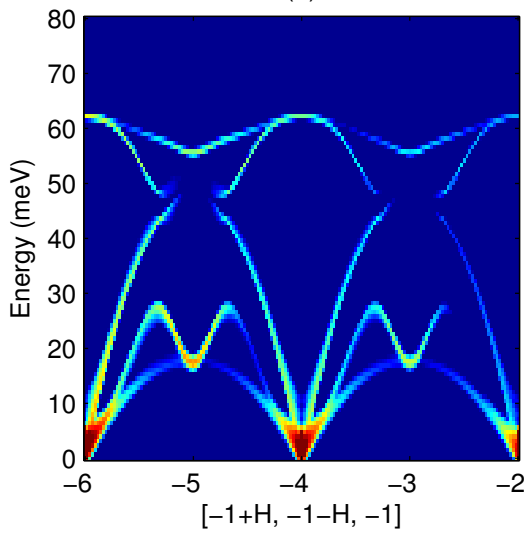

Figure 7: Images of slice 3: subplot (a) is the experimental data; subplot (b) is the simulation of the theoretical model given the initial guess of the parameters; subplot (c) is the simulation of the theoretical model after optimizing the first 5 parameters in the first optimization loop; subplot $(\mathrm{d})$ is the simulation of the theoretical model obtained by using the optimal parameter. $H$ is the reciprocal lattice unit.

optimization problem, we search the entire parameter space by testing random chosen parameters following a hierarchical manner. To address the large data comparison problem, we provided a data decomposition method which allows us to compare theoretical results with a portion of data at each time and then combine the comparison result of each portion. Numerical experiments show the effectiveness and accuracy of our algorithm. In the future, we plan to study more complicated material with real experimental data receive from detectors and apply our algorithm to material science research.

\section{Acknowledgements}

Acknowledges support by the U.S. Department of Energy, Office of Science, Basic Energy Sciences, Materials Sciences and Engineering Division, through the Office of Science Early Career Research Program and Advanced Scientific Computing Research, through the ACUMEN project. 


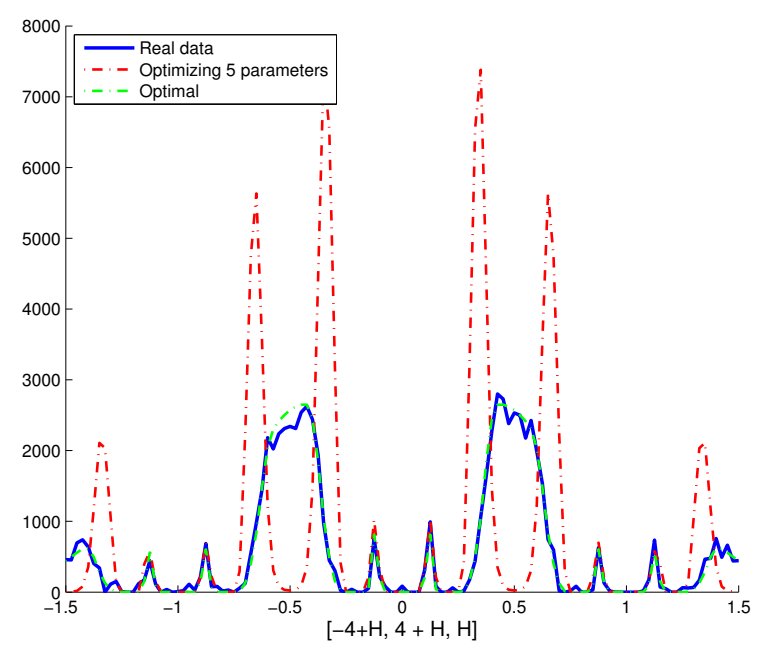

Figure 8: Intensity comparison of slice 1

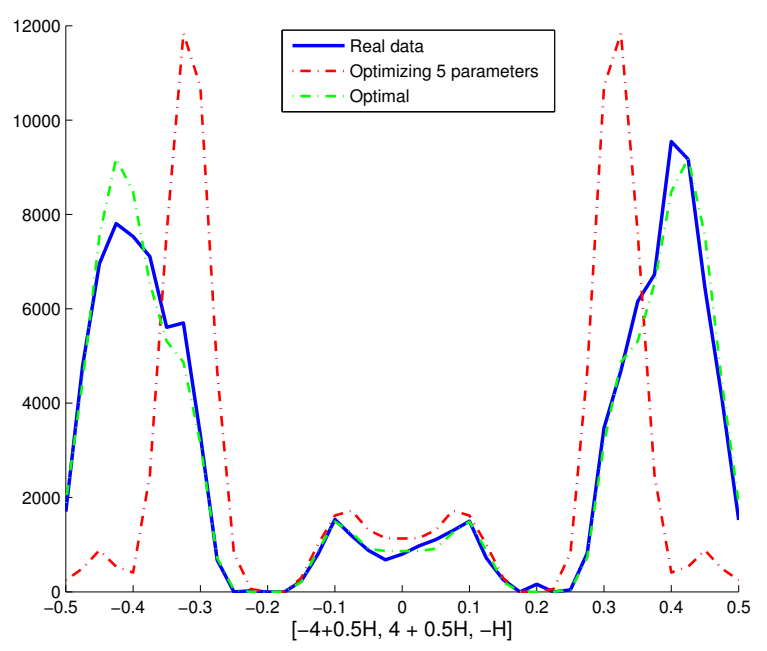

Figure 9: Intensity comparison of slice 2

\section{References}

[1] S. Lovesey, Theory of Neutron Scattering from Condensed Matter, Vol. 1, Oxford University Press, Oxford, 1984.

[2] D. Price, K. Skold, Neutron Scattering, Academic Press, New York, 1986.

[3] G. Squires, Introduction to the Theory of Thermal Neutron Scattering, Cambridge University Press, Cambridge, 1978. 


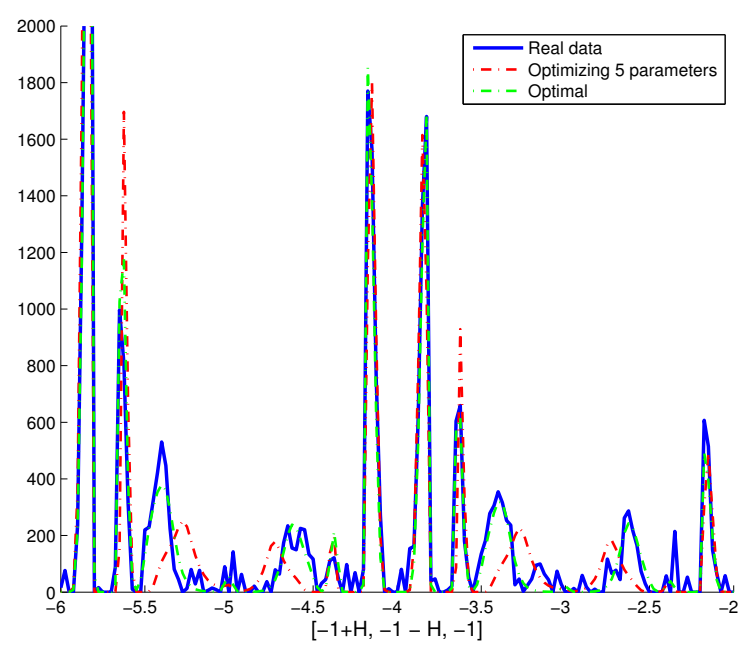

Figure 10: Intensity comparison of slice 3

Table 1: Top ranked weights (iteration step 8)

\begin{tabular}{|c|c|c|c|c|}
\hline Rank & Slice 1 & Slice 2 & Slice 3 & Overall \\
\hline 1 & $2.86 \%$ & $0.60 \%$ & $5.39 \%$ & $25.86 \%$ \\
2 & $2.65 \%$ & $0.59 \%$ & $4.71 \%$ & $16.43 \%$ \\
3 & $2.65 \%$ & $0.58 \%$ & $4.39 \%$ & $10.81 \%$ \\
4 & $2.34 \%$ & $0.58 \%$ & $4.07 \%$ & $9.88 \%$ \\
5 & $2.34 \%$ & $0.58 \%$ & $3.98 \%$ & $5.55 \%$ \\
6 & $2.31 \%$ & $0.58 \%$ & $3.93 \%$ & $5.36 \%$ \\
7 & $2.23 \%$ & $0.57 \%$ & $3.24 \%$ & $4.07 \%$ \\
8 & $2.14 \%$ & $0.57 \%$ & $3.13 \%$ & $3.32 \%$ \\
9 & $2.14 \%$ & $0.56 \%$ & $3.10 \%$ & $2.95 \%$ \\
10 & $2.14 \%$ & $0.56 \%$ & $2.87 \%$ & $2.72 \%$ \\
11 & $2.13 \%$ & $0.56 \%$ & $2.28 \%$ & $2.34 \%$ \\
12 & $2.13 \%$ & $0.56 \%$ & $2.18 \%$ & $1.55 \%$ \\
13 & $2.10 \%$ & $0.56 \%$ & $2.07 \%$ & $1.44 \%$ \\
14 & $1.96 \%$ & $0.55 \%$ & $2.04 \%$ & $0.95 \%$ \\
15 & $1.93 \%$ & $0.55 \%$ & $2.00 \%$ & $0.78 \%$ \\
\hline
\end{tabular}

[4] P. Brüesch, Phonons: Theory and Experiments I - Lattice Dynamics and Models of Interatomic Forces, Vol. 34, Springer Series in Solid-State Sciences, New York, 1982.

[5] O. Delaire and C. Stassis, Phonon Studies, in Characterization of Materials, Wiley (2012).

[6] J. Pedersen, D. Posselt, K. Mortensen, Analytical treatment of the resolution function for small-angle scattering, Journal of Applied Crystallography 23 (1990) 321-333.

[7] W. Schmidt, D. Sieger, H. Tietze-Jaensch, R. Geick, A new fast four-dimensional convolution algorithm for the interpretation of neutron scattering data, Nuclear Instruments and Methods in Physics Research A316 (1992) 364-387. 
Table 2: Top ranked weights (iteration step 16)

\begin{tabular}{|c|c|c|c|c|}
\hline Rank & Slice 1 & Slice 2 & Slice 3 & Overall \\
\hline 1 & $0.41 \%$ & $0.19 \%$ & $0.51 \%$ & $2.05 \%$ \\
2 & $0.41 \%$ & $0.19 \%$ & $0.49 \%$ & $1.93 \%$ \\
3 & $0.40 \%$ & $0.19 \%$ & $0.49 \%$ & $1.87 \%$ \\
4 & $0.40 \%$ & $0.19 \%$ & $0.48 \%$ & $1.81 \%$ \\
5 & $0.40 \%$ & $0.19 \%$ & $0.48 \%$ & $1.73 \%$ \\
6 & $0.40 \%$ & $0.19 \%$ & $0.48 \%$ & $1.56 \%$ \\
7 & $0.39 \%$ & $0.19 \%$ & $0.47 \%$ & $1.52 \%$ \\
8 & $0.39 \%$ & $0.19 \%$ & $0.47 \%$ & $1.52 \%$ \\
9 & $0.39 \%$ & $0.19 \%$ & $0.47 \%$ & $1.51 \%$ \\
10 & $0.39 \%$ & $0.19 \%$ & $0.47 \%$ & $1.50 \%$ \\
11 & $0.38 \%$ & $0.19 \%$ & $0.47 \%$ & $1.48 \%$ \\
12 & $0.38 \%$ & $0.19 \%$ & $0.47 \%$ & $1.41 \%$ \\
13 & $0.38 \%$ & $0.19 \%$ & $0.46 \%$ & $1.36 \%$ \\
14 & $0.38 \%$ & $0.19 \%$ & $0.46 \%$ & $1.32 \%$ \\
15 & $0.37 \%$ & $0.19 \%$ & $0.45 \%$ & $1.12 \%$ \\
\hline
\end{tabular}

[8] W. Ulmer, Inverse problem of linear combinations of gaussian convolution kernels (deconvolution) and some applications to proton/photon dosimetry and image processing, Inverse Problems 26 (2010) 085002.

[9] L. Greengard, J. Strain, The fast gauss transform, SIAM Journal on Scientific and Statistical Computing 12 (1991) 79-94.

[10] C. Yang, R. Duraiswami, N. Gumerov, Improved fast gauss transform and efficient kernel density estimation, Proceedings of Ninth IEEE International Conference on Computer Vision 1 (2003) 664671.

[11] M. Rostami, Compressed Sensing with Side Information on the Feasible Region, Sprinter Briefs in Electrical and Computer Engineering, Springer, 2014.

[12] X. Liu, Z. Wen, Y. Zhang, Limited memory block krylov subspace optimization for computing dominant singular value decompositions, SIAM Journal on Scientific Computing 35 (2013) 1641-1668.

[13] M. D. McKay, R. J. Beckman, W. J. Conover, A comparison of three methods for selecting values of input variables in the analysis of output from a computer code, Technometrics 21 (2) (1979) pp. 239-245.

URL http://www . jstor . org/stable/1268522

[14] O. Delaire, J. Ma, K. Marty, A. F. May, M. A. McGuire, M.-H. Du, D. J. Singh, A. Podlesnyak, G. Ehlers, M. Lumsden, and B. C. Sales, Nature Materials 10, 614 (2011).

[15] C.W. Li, J. Ma, H.B. Cao, A.F. May, D.L. Abernathy, G. Ehlers, C. Hoffmann, X. Wang, T. Hong, A. Huq, O. Gourdon, and O. Delaire, Phys. Rev. B 90, 214303 (2014). 
[16] O. Delaire, I. I. Al-Qasir, A. F. May, C. W. Li, B. C. Sales, J. L. Niedziela, J. Ma, M. Matsuda, D. L. Abernathy, T. Berlijn, Phys. Rev. B 91, 094307 (2015).

[17] D. L. Abernathy, M. B. Stone, M. J. Loguillo, M. S. Lucas, O. Delaire, X. Tang, J.Y.Y. Lin, and B. Fultz, Rev. Sci. Instrum. 83, 15114 (2012).

[18] Ehlers, G and Podlesnyak, A A and Niedziela, J L and Iverson, E B and Sokol, P E, Rev. Sci. Instrum. 82, 8 (2011).

[19] SIMPHONIES: software for the simulation of phonon inelastic scattering, O. Delaire et al. to be published. 